\title{
DETECTION AND VERIFICATION FOR THE PRESENCE OF STRESS BY MEANS OF VOICE ANALYSIS AND NEURAL NETWORKS
}

\author{
Stefan HAdJitodorov ${ }^{*}$ and BOYAN BOYANOV \\ Institute on Biophysics and Biomedical Engineering, \\ Bulgarian Academy of Sciences, \\ Acad. G. Bonchev St, Block 105, 1113 Sofia, Bulgaria, \\ e-mail: STHADJ@BAS.BG
}

\begin{abstract}
An approach for verification and detection the presence of stress by means of speech analysis and neural networks is proposed. The speech parameters are evaluated by a procedure including periodicity/aperiodicity separation, robust pitch period (To) evaluation, elimination of wrongly calculated To, calculation of the index of microprosodic variation of $T o$, calculation of autocorrelation cepstral coefficients, evaluation of the group delay function by different procedures for low and for high pitched voices. The stress verification and stress detection are performed by neural networks introduced by the authors - Prototype Distribution neural Maps (PDM). Stress detection: The speaker's parameters in normal situation and under stress are modeled by two PDMs - PDM $M_{\text {norma }}$ and $P D M_{\text {stress. }}$. Stress verification: For every speaker personal $P D M_{\text {norma }}$ and $P D M_{\text {stress }}$ are trained. During the stress verification and detection similarities between the PDM of the speaker under evaluation and $P D M_{\text {norma }}\left(\right.$ Sim $\left._{\text {norma }}\right)$ and PDM $M_{\text {stress }}\left(\right.$ Sim $\left._{\text {stress }}\right)$ are calculated. The stress detection and verification are realized by analysis the ratio $\operatorname{Sim}_{\text {norma }} /$ Sim $_{\text {stress }}$ using specific rules.

Keywords: stress detection and verification, speech analysis, neu-
\end{abstract} ral networks.

\section{INTRODUCTION}

The speech generation is a complex act involving the central nervous system, the vocal tract, the vocal folds, the respiratory system, etc. It is known that the stress affects the functioning of the above mentioned systems. As a result specific changes in the speech may be expected. This is the reason for

${ }^{*}$ Corresponding author.

DOI: 10.7546/EngSci.LVII.20.04.01

Engineering Sciences, LVII, 2020, No. 4 
many studies in this field, part of them referred here $[1 \div 36]$. An important research in [5] has been carried out, where 200 parameters were analyzed and 10000 statistical tests were performed for evaluation the correlation between the stress and changes in some speech parameters. The above mentioned researches have shown that the following speech parameters are affected by the stress:

- pitch period $(T o)$;

- statistics of pitch period;

- spectral shifts of the first three formants;

- intensity of the signal;

- durations of pauses and periods of phonations;

- spectral characteristics;

- cepstral characteristics;

- low frequency modulation of the fundamental frequency $(F o)$.

The above mentioned researches are based on analysis of the speech of people in the following situations: (i) pilots in extremal situations; (ii) actors simulating different emotions; (iii) singers under stress (during presentation), etc.

The research team of Duke University has developed an excellent tool for evaluation the effectiveness of the approaches and methods for stress detection - a speech under stress database "SUSAS" $[5,6]$ including practically all the emotions.

Here an approach for automatic stress detection and for verification of the presence of stress by means of speech analysis and neural networks is proposed.

\section{EVALUATION OF THE SPEECH PARAMETERS}

In order to minimize the errors during the speech parameters evaluation, the following procedure for speech analysis is proposed and used:

\subsection{Segmentation of the speech signal}

The quantized signal is divided into segments with length three To by means of a Hamming window. The duration of the segments is dynamically adapted to 3 To (using To from the previous segment), because our experiments [37] have shown that such segment length is optimal for To evaluation.

The overlapping between the segments is two pitch periods in order to analyze more completely the dynamics of the speech parameters.

The length of the first segment is $30 \mathrm{~ms}$ in order to assure that the window contains at least 2 To. 


\subsection{Periodicity/aperiodicity separation}

The Periodicity/Aperiodicity Separation (PAS) is very important for correct To detection, because errors in PAS will produce drastic errors in To. In order to minimize the number of errors in PAS the detector proposed in [38] is implemented, because it is characterized by:

1. Parallel analysis of the speech in time, spectral and cepstral domains. In this way different characteristics of the signal in these domains are used and the signal is analyzed more completely and from different viewpoints.

2. Realization of robust PAS by means of MultiLayer Perceptron (MLP) neural network. As a result the accuracy is improved, because the MLP is characterized by good discriminant capabilities and high classification power.

In order to minimize the influence of the noisy components the aperiodic segments are eliminated.

\subsection{Pitch period $(T o)$ evaluation}

In order to evaluate correctly To the robust hybrid pitch detector [39] is used. This method is implemented, because it has the following useful properties:

a) Rejects practically most of the segments, where To is wrongly evaluated (experimental research over 200 speakers [37] when the signal is preprocessed by the PAS detector [38]. However it eliminates up to $1 \%$ of the voiced segments. The loss of these segments may be tolerated, because for all the speakers (in our database) the analyzed sentences are relatively longer than 6 seconds and contain more than 200 segments;

b) Evaluates correctly To from clean, noisy and telephone speech;

c) Realizes parallel analysis of the speech signal in temporal, spectral and cepstral domains;

d) Evaluates the pitch period by means of logical analysis of the results from these three domains.

\subsection{Elimination of wrongly calculated $T o$}

The elimination of wrongly calculated values of $T o$ is realized by means of the following algorithm:

Step 1. Formation of segments containing 3 To.

Step 2. For every segment (containing $3 T o$ ) To is calculated again $\left(T o_{n e w}\right)$ using the same detector [39].

Step 3. Comparison of the value of $T o_{\text {new }}$ with the value of $T o$ obtained from the previous analysis:

Engineering Sciences, LVII, 2020, No. 4 
if

$$
\left|T o_{\text {new }}-T o\right|>0.3 T o,
$$

the segment is eliminated.

\subsection{Calculation of the mean pitch period $\left(T o_{m}\right)$ and its index of microprosodic variation}

Many experimental researches $[1,5,10,15,17 \div 19,21,22,26,31,32]$ have shown that $T o$ is significantly affected by the stress. To evaluate and analyze this property of $T o$ for every p-th segment (containing $3 \mathrm{To}$ ) the mean pitch period $\left(T o_{m}(p)\right)$ and its Index of MicroProsodic Variation $(I M V(p))$ are calculated. The $I M V$ is used, because several experiments in the research [18] have shown that this parameter is correlated with the presence of stress. The $I M V$ is calculated by means of the following formula described in [18]:

$$
I M V(p)-T o_{\text {mid }}(p) /\left\{\left(T o_{\text {beg }}(p)+T o_{\text {end }}(p)\right) / 2\right\},
$$

where $T o_{b e g}(p)$ is the value of $T o$ at the beginning of $p$-th segment; $T o_{\text {mid }}(p)$ - value of $T o$ in the middle of $p$-th segment; $T o_{\text {end }}(p)$ - value of $T o$ at the end of $p$-th segment.

\subsection{Cepstral analysis (over the voiced segments)}

The experimental researches $[5 \div 8,29]$ have revealed that the standard LPderived cepstral coefficients $(c(n))$ and especially the autocorrelation cepstral coefficients $(a c c(k))$ (introduced for stress detection by Professor Hansen) are very informative for stress detection. For that reason in the proposed approach the $(\operatorname{acc}(k))$ are used. In order to minimize the influence of the noisy components the $(c(n))$ are calculated for voiced segments. The cepstral analysis is carried out by means of the standard procedure LPC analysis by means of the autocorrelation method [40] and then the first 8 LPC coefficients are calculated. The number of $(c(n))$ is 8 , because the experimental research [29] has made known that the first 7 coefficients are the most informative for stress detection. From these $(c(n))$ are calculated the $(\operatorname{acc}(k))$ by means of the formulae described and discussed in detail in [29]. In the proposed approach the value of the correlation lag is 1 and the length of the correlation window is 5 , because in our database most of the periodic portions of speech signal contained $4-5$ windows. 


\subsection{Evaluation of the group delay function (for the voiced segments)}

In $[1,9,10,17 \div 19,21,22]$ it has been shown that the stress causes changes in the formant structure. However the estimation of the formants is a difficult problem not yet completely solved. That is why the changes in the formant structure are analyzed by evaluation and analysis of the Group Delay Function $(G D F)(G D F$ is the negative derivative of the phase spectrum). The $G D F$ is used for approximation of the formant structure, because the $G D F$ has the following useful properties [41]:

1. The $G D F$ is proportional to the squared magnitude response near resonances (formants) and approaches zero asymptotically for frequencies away from the frequency of the resonator. In this way the formants are represented by distinct and sharp peaks in the $G D F$.

2 . The vocal tract may be represented by a cascade of resonators. The GDF of such system is the sum of GDFs of these resonators. As a result the influence of one resonator to another is minimized - even closely spaced formants are represented in the $G D F$ by separated peaks.

The main problems in calculation of the $G D F$ are:

1. The phase function is warped by the presence of zeros near the unit circle and the signal windowing prior the spectral analysis [41].

2. Most of the methods of phase unwarping do not yield satisfactory results [42].

3. The spectral resolution in the $G D F$ is decreased for medium and high pitched voices ( $T o<5 \mathrm{~ms}$ and $F_{O}>200 \mathrm{~Hz}$ ), when $G D F$ is calculated from $c(n)$. This is due to the short To minimizing the number of $c(n)$ and respectively the number of $G D F$ coefficients $(g d f(i))$. For shorter than 5 ms $T o$ the spectral resolution will be less than $262 \mathrm{~Hz}$, because in our experiments the sampling rate is $21 \mathrm{KHz}$.

4. Distortions (represented in most of the cases by extra peaks) caused by the influence of the glottal source.

In order to solve these problems and to guarantee spectral resolution higher than in the standard wide band sonogram used for formant analysis (here 262 $\mathrm{Hz}$ ), the following approach for $G D F$ calculation is proposed and used:

\subsubsection{Analysis of low pitched voices}

For such voices the $G D F$ is calculated indirectly (without phase calculation and unwarping) from $c(n)$ - detailed proof is given in [41]. In order to minimize the influence of the glottal source only the cepstral coefficients 
$\left(c_{v}(n)\right)$ corresponding to the vocal tract are separated by means of liftering. The length $(L)$ of the lifter is less than To. The value of $L$ is equal to $0.8 T o$, because our experiments [37] have shown that such length is sufficient for suppression of the influence of the glottal source. The $G D F$ is calculated by means of the formula described in [41]:

$$
G D F(i)=\frac{2 \pi}{N} \sum_{n=0}^{N-1} n c_{v}(n) \cos \left(\frac{2 \pi n i}{N}\right),
$$

where $N$ is the number of cepstral coefficients, used for $G D F$ calculation.

The value of $N$ is limited to 80 , because the shortest $T o$ used for calculation of the $(g d f(i))$ from $c(n)$ is $5 \mathrm{~ms}$ and the sampling rate is $21 \mathrm{KHz}$.

\subsubsection{Analysis of high pitched voices}

For such voices the $G D F$ is calculated by means of the following procedure described in [43]:

a) transformation of the voiced speech into minimum phase signal;

b) direct calculation of the phase spectrum form this minimum phase signal;

c) calculation of the first derivative of the phase spectrum - the GDF.

Unfortunately for the high pitched voices the influence of the glottal source is not suppressed, however in most of the practical cases male voices are analyzed, generally characterized with low values of the pitch period.

The first $S$ numbers of $(g d f(i)) \quad($ for $i=1, \ldots S)$ are used like a feature vector representing the formant structure. The value of $S$ is determined on the basis of bass band of the Cockpit Voice Recorders (CVR), because one of the main applications of the proposed approach will be aircraft speech communication monitoring. The bass band of the CVR is $150-5000 \mathrm{~Hz}$ (Ritz, 1994) and to cover this spectral range the value of $S$ is 19, because in our experiments the sampling rate is $21 \mathrm{KHz}$ and the resolution in the GDF is $262 \mathrm{~Hz}$.

\section{STRESS DETECTION BY MEANS OF NEURAL NETWORKS}

The following input vectors $(y(t))$ for neural network (used for stress detection) are formed for every $\mathrm{p}$-th (for $p=1, \ldots P$ ) voiced segment:

1. The mean pitch period $\left(T o_{m}(p)\right)$ and its index of microprosodic variation $(I M V(p))$;

2. The first 8 acc $(i) \quad($ for $i=1, \ldots 8)$; 
3. The first $19(g d f(i)) \quad($ for $i=1, \ldots 19)$.

In order to increase the accuracy of stress detection, the speech parameters of the speakers in the two psycho-emotional situations (classes - normal and stress) are modeled by means of two neural maps named Prototype Distribution Maps (PDMs). The PDM was proposed and described in detail in [44]. The $P D M s$ are used because they have the following useful properties [44, 45]:

1. The $P D M$ neurons try to imitate the probability density function $(p d f)$ of the input signals, whatever complex the form of the $p d f$ is. This property is due to the fact that the $P D M$ is formed on the basis of the trained Self-Organizing Map of Kohonen $(S O M)$ [46].

2. The $P D M$ allows dimensionality reduction - the two-dimensional $S O M$ with n-dimensional weight vectors is transformed into a two-dimensional map with one dimensional weight vectors (named frequencies of activation).

3. Less significant neurons are eliminated by filtering of the $P D M$.

\subsection{Stress detection}

\subsubsection{Training stage - formation of two sample PDMs}

Step 1. Using the input vectors of the speakers in normal situation $\left(y_{\text {norma }}(t)\right)$ and under stress $\left(y_{\text {stress }}(t)\right)$ one joint $S O M$ of size $Q \times Q$ (here $Q=15)$, named $S O M_{\text {joint }}$, is formed. This $S O M_{\text {joint }}$ approximates the overall probability density function $(p d f)$ of all the input vectors.

Step 2. Tuning of the $S O M_{\text {joint }}$. In order to increase the accuracy of modeling of the input vectors by means of $S O M_{\text {joint }}$ a long term fine tuning of $S O M_{\text {joint }}$ by means of the Learning Vector Quantisation algorithm - LVQ (detailed description in [46] is done. The number of iterations is not limited and the tuning process continues until optimal tuning of $99 \%$ from the neurons is reached.

Step 3. Formation of two sample PDMs ( $P D M_{\text {norma }}$ and $\left.P D M_{\text {stress }}\right)$ for the two classes (NORMA and STRESS). The following procedure proposed in [44] is used:

1. The input vectors $y_{\text {norma }}(t)$ and $y_{\text {stress }}(t)$ are passed separately again through the already trained $S O M_{\text {joint }}$. As a result for the two classes for every neuron the activation frequency $\left(f_{i, j}, i, j=1, \ldots Q\right)$ is obtained. These $f_{i, j}$ are normalized by dividing them by the number of all the feature vectors in the given utterance/s. The activation frequency represents the number of input vectors which have activated this neuron, according 
to the minimum of Euclidean distance. These two maps in fact represent the probability of distribution of input vectors.

2. These $P D M s$ are filtered in order to eliminate sporadically activated locations (noncharacteristic neurons) by means of the filtering procedure described in [44].

\subsubsection{Detection the presence of stress}

The following procedure for stress detection is proposed and used:

1. The data vectors $y_{x}(t)$ of the speaker under evaluation are passed through the already formed $S O M_{\text {joint }}$. As a result the $P D M_{x}$ is obtained for this speaker.

2. Filtering of $P D M_{x}$ by means of the same procedure and same value of the filter coefficient as on step 3 during the training.

3. The similarities between $P D M_{x}$ and $P D M_{\text {norma }} \quad\left(\right.$ Sim $\left._{\text {norma }}\right)$ and $P D M_{x}$ and $P D M_{\text {stress }}\left(\right.$ Sim $\left._{\text {stress }}\right)$ are evaluated by means of a cross-correlation type measure proposed in [44]. The $S_{i m_{n o r m a}}$ and $S_{i m}$ stress are calculated by the formulae:

$$
\begin{aligned}
\operatorname{Sim}_{\text {norma }} & =\sum_{i=1}^{q} \sum_{j=1}^{q}\left(f_{i, j}^{N o r} \cdot f_{i, j}^{X}\right)\left(d+\left|f_{i, j}^{N o r}-f_{i, j}^{X}\right|\right)^{-1}, \\
\text { Sim }_{\text {stress }} & =\sum_{i=1}^{q} \sum_{j=1}^{q}\left(f_{i, j}^{S t r} \cdot f_{i, j}^{X}\right)\left(d+\left|f_{i, j}^{S t r}-f_{i, j}^{X}\right|\right)^{-1},
\end{aligned}
$$

where $f_{i, j}^{N o r}$ is the frequency of activation of a neuron in $P D M_{n o r m a} ; f_{i, j}^{S t r}$ - frequency of activation of a neuron in $P D M_{\text {stress }} ; f_{i, j}^{X}$ - frequency of activation of a neuron in $P D M_{x} ; d$-experimentally determined constant $0<d<1$. The values of $d=0.1$, because our experiments have shown that $d=0.1$ is optimal for separation of two classes in the case of laryngeal pathology detection [47] and speaker recognition [37, 44].

4. Calculation of the ratio $\operatorname{Sim}_{\text {norma }}$ - to - Sim stress:

$$
R N S=\operatorname{Sim}_{\text {norma }} / \text { Sim }_{\text {stress }} .
$$

5. Stress detection.

In order to realize robust stress detection the following rules are proposed and used:

Case 1. Classification of the speaker under evaluation in class NORMA (absence of stress) if:

$$
R N S>1+k_{n o r}
$$


where $k_{n o r}$ is the experimentally determined coefficient $k_{\text {nor }}<1$;

Case 2. Classification of the speaker under evaluation in class STRESS (stress detection) if:

$$
R N S<1-k_{\text {stress }},
$$

where $k_{\text {stress }}$ is the experimentally determined coefficient $k_{\text {stress }}<1$.

In order to avoid classification of a person under stress as a speaker in normal situation the following inequality is introduced:

$$
k_{\text {stress }}<k_{n o r}<1
$$

Case 3. Refusal from classification (no classification is performed):

$$
\left(1-k_{\text {stress }}\right)<=R N S<=\left(1+k_{\text {nor }}\right) .
$$

The optional values (for our database) of $k_{\text {nor }}$ and $k_{\text {stress }}\left(k_{n o r}=0.2\right.$ and $\left.k_{\text {stress }}=0.1\right)$ are obtained during the experimental research.

\subsubsection{Closed set and open set stress detection}

In analogy with speaker recognition the stress detection may be performed over an open or closed set. The closed set stress detection is performed only over the speakers from the database - feature vectors of these speakers are used on the training stage. The open set stress detection may be performed over unknown speakers too. In the present study open and closed set stress detection have been carried out.

\subsection{Verification for the presence of stress}

The following procedure for stress verification is proposed and used:

\subsubsection{Training stage - formation of two personal sample PDMs}

Step 1. Using the input feature vectors of every $\mathrm{j}$-th speaker separately in normal situation $\left(y_{\text {norma }}(t)\right)$ and under stress $\left(y_{\text {stress }}(t)\right)$ one personal $S O M_{\text {joint }}(j)$ of size $Q \times Q$ (here $\left.Q=15\right)$ is formed.

Step 2. Tuning of the $S O M_{\text {joint }}(j)$ by means of the procedure used on stress detection (Section 3.1).

Step 3. Formation of two personal samples $P D M s\left(P D M_{\text {norma }}(j)\right.$ and $P D M_{\text {stress }}(j)$ for every speaker by means of the procedure used on stress detection.

Engineering Sciences, LVII, 2020, No. 4 


\subsubsection{Verification for the presence of stress}

The following procedure for stress verification is proposed and used:

1. The data vectors of the speaker (only for speaker from the database, disposing with their individual $S O M$ and $P D M s$ ) under evaluation are passed through its individual $S O M_{\text {joint }}(j)$. As result the $P D M_{x}(j)$ is obtained for this speaker.

2. Filtering of $P D M_{x}(j)$ by means of the same procedure and same value of the filter coefficient as that during the training.

3. The similarities between $P D M_{x}(j)$ and its personal $P D M_{n o r m a}(j)$ $\left(\operatorname{Sim}_{\text {norma }}\right)$ and $P D M_{x}(j)$ and its personal $P D M_{\text {stress }}(j) \quad\left(\operatorname{Sim}_{\text {stress }}\right)$ are evaluated by means of the procedure used on stress detection.

4. Calculation of the ratio $S i m_{n o r m a}$ - to - Sim stress by means of the procedure used on stress detection.

5. Robust stress verification by means of the above used rules for stress detection.

\section{EXPERIMENTAL RESEARCH}

\subsection{Subjects and speech material}

The experiments were carried out in the studios of four private radio stations in Sofia, Bulgaria. The speech of 30 radio speakers in normal communication and "in air" was analyzed. Two sentences (in Bulgarian): "My name is ....." and "We start the emission today with the international news" pronounced in normal communication and "in air" were analyzed.

The range of the number of voiced segments (respectively the input vectors) were from 240 to 570 per sentence.

\subsection{Equipment}

The standard audio board "Sound Blaster" was used with sampling rate of $21 \mathrm{KHz}$ and resolution of $16 \mathrm{bit}$. The microphone was at a distance of about $30 \mathrm{~cm}$ from the mouth of the subjects. The voice signals were quantified and stored directly into the computer memory in order to avoid distortions caused by tape recorders. The tape recorders introduce distortions in the values of the calculated voice parameters, as shown in [48]. 


\subsection{Stress detection}

\subsubsection{Closed set stress detection}

During the training stage the sentence "We start the emission today with the international news", uttered in normal situation and under stress ("in air") by all the radio speakers was used. The test was realized over the sentence "My name is ..." uttered in normal communication and "in air" by all the speakers.

\subsubsection{Open set stress detection}

During the training stage the speech of 15 radio speakers pronouncing the two sentences was used. The tests were performed over the remaining 15 radio speakers, pronouncing the same two sentences.

\subsection{Verification for the presence of stress}

During the training stage using the sentence "We start the emission today with the international news" in normal communication and under stress ("in air") for every speaker two personal PDMs $P D M_{\text {norma }}(j)$ and $P D M_{\text {stress }}(j)$ were trained (i.e. $60 P D M s$ are formed). The test was realized over the sentence "My name is ...".

\section{RESULTS}

The optional values (for our database) of $k_{n o r}$ and $k_{\text {stress }}\left(k_{n o r}=0.2\right.$ and $\left.k_{\text {stress }}=0.1\right)$ are obtained during the experimental research. For the optimal values of $k_{n o r}$ and $k_{\text {stress }}$ the following results were obtained:

\subsection{Stress detection}

\subsubsection{Closed set stress detection}

The number of errors in stress detection for closed set in function of $k_{\text {stress }}$ and $k_{n o r}$ is given in Table 1 . One erroneous classification and no classification (decision) for 4 speakers were observed.

Engineering Sciences, LVII, 2020, No. 4 
Table 1. Numbers of wrong stress detection (closed set) in function of $k_{\text {stress }}$ and $k_{n o r}$

\begin{tabular}{|c|c|c|c|c|}
\hline $\begin{array}{c}\text { Values } \\
\text { of } k_{\text {nor }}\end{array}$ & $\begin{array}{c}\text { Values } \\
\text { of } k_{\text {stress }}\end{array}$ & $\begin{array}{c}\text { Number } \\
\text { of errors }\end{array}$ & $\begin{array}{c}\text { Number } \\
\text { of errors }\end{array}$ & $\begin{array}{c}\text { Number } \\
\text { of decisions }\end{array}$ \\
\hline 0.05 & 0.025 & Norma as Stress & Stress as Norma & \\
\hline 0.10 & 0.050 & 2 & 3 & 0 \\
\hline 0.20 & 0.100 & 2 & 0 & 1 \\
\hline 0.30 & 0.200 & 0 & 0 & 4 \\
\hline
\end{tabular}

\subsubsection{Open set stress detection}

The number of errors in stress detection for open set in function of $k_{\text {stress }}$ and $k_{n o r}$ is given in Table 2 . Two erroneous classifications and no classification (decision) for 7 speakers were observed.

Table 2. Numbers of wrong stress detection (open set) in function of $k_{\text {stress }}$ and $k_{n o r}$

\begin{tabular}{|c|c|c|c|c|}
\hline $\begin{array}{c}\text { Values } \\
\text { of } k_{\text {nor }}\end{array}$ & $\begin{array}{c}\text { Values } \\
\text { of } k_{\text {stress }}\end{array}$ & $\begin{array}{c}\text { Number } \\
\text { of errors }\end{array}$ & $\begin{array}{c}\text { Number } \\
\text { of errors }\end{array}$ & $\begin{array}{c}\text { Number } \\
\text { of decisions }\end{array}$ \\
\hline & & Norma as Stress & Stress as Norma & \\
\hline 0.05 & 0.025 & 4 & 5 & 0 \\
\hline 0.10 & 0.050 & 3 & 4 & 2 \\
\hline 0.20 & 0.100 & 1 & 1 & 10 \\
\hline 0.30 & 0.200 & 0 & 0 & \\
\hline
\end{tabular}

\subsection{Verification for the presence of stress}

The number of errors in stress verification in function of $k_{\text {stress }}$ and $k_{n o r}$ is given in Table 3. No erroneous classification was detected, however for 3 speakers no classification has been realized.

Table 3. Numbers of wrong stress verification in function of $k_{\text {stress }}$ and $k_{n o r}$

\begin{tabular}{|c|c|c|c|c|}
\hline $\begin{array}{c}\text { Values } \\
\text { of } k_{\text {nor }}\end{array}$ & $\begin{array}{c}\text { Values } \\
\text { of } k_{\text {stress }}\end{array}$ & $\begin{array}{c}\text { Number } \\
\text { of errors }\end{array}$ & $\begin{array}{c}\text { Number } \\
\text { of errors }\end{array}$ & $\begin{array}{c}\text { Number } \\
\text { of decisions }\end{array}$ \\
\hline & & Norma as Stress & Stress as Norma & \\
\hline 0.1 & 0.05 & 1 & 2 & 0 \\
\hline 0.2 & 0.10 & 0 & 0 & 3 \\
\hline 0.3 & 0.20 & 0 & 0 & 6 \\
\hline
\end{tabular}




\section{CONCLUSIONS}

The results obtained show that the proposed approach could be applied for detection the presence of stress and for robust verification of presence of stress. When reporting about these results however we have to take into account the fact that the speech of 30 professional radio speakers has been analyzed. In the same time, it is important to note that 18 of these persons are working less than one year like professional radio speakers, i.e. most of them are persons with insufficient professional experience. Having in mind that, we can conclude these encouraging results may be a basis for our future researches in the field of stress detection and verification using voice analysis and various neural networks.

\section{Glossary of the abbreviations used in the text:}

To Pitch period (the period of the voiced speech signal);

$P A S$ Periodicity/aperiodicity separation;

$I M V$ Index of microprosodic variation;

GDF Group delay function;

$S O M \quad$ Self-organizing map;

$P D M$ Prototype distribution map;

$L V Q \quad$ Learning vector quantization algorithm;

$M L P \quad$ Multilayer perceptron neural network.

\section{REFERENCES}

[1] O. Asparoukhov and B. Boyanov, Computer analysis of the speech of patient under preoperative stress, Acustica (1994) 80 412-414.

[2] M. Brenner, H. Branscomb, and G. Schwartz, Psychological stress evaluator, two tests of a vocal measure, Psychophysiology (1979) 16 351-357.

[3] D. Cairns and J. Hansen, Nonlinear analysis and classification of speech under stressed conditions, J. Acoust. Soc. Am. (1994) 96 (6) 3392-3400.

[4] P. Greasley, J. Setter, M. Waterman, G. Sherrard, P. Roach, S. ARnfield, AND D. HoRTon, Representation of prosodic and emotional features in spoken language database, in: Proc. International Conference on Spoken Language Processing, 1995, 1, pp. 242-245.

[5] J. Hansen, Analysis and compensation of speech under stress and noise for environmental robustness in speech recognition, in: Proc. ESCA-NATO Workshop on "Speech under stress", Lisbon, Portugal, 1995, pp. 91-98. 
[6] J. Hansen, Morphological constrained feature enhancement with adaptive cepstral compensation (MCE-ACC) for speech recognition in noise and Lombard effect, IEEE Trans. Speech and Audio Processing (1994) 2 598-614.

[7] J. Hansen And M. Clements, Source generator equalization and enhancement of spectral properties for robust speech recognition in noise and stress, IEEE Trans. Speech and Audio Processing (1995) 3 407-415.

[8] J. Hansen And B. Womack, Feature analysis and neural network based classification of speech under stress, IEEE Trans. Speech and Audio Processing (1996) 4 304-313.

[9] M. Hecker, K. Stevens, G. von Bismark, and C. Williams, Manifestations of task-induced stress in the acoustic speech signal, J. Acoust. Soc. Am. (1968) 44 993-1001.

[10] H. Hollien, Vocal indicators of psychological stress, Ann. New York Acad. Sciences (1980) 347 47-68.

[11] W. Hicks and H. Hollien, The reflection the stress in voice-1: Understanding the basic correlates, in: Proc. Carnahan Conf. on Crime Countermeasures, Lexington, USA, 13-15 May, 1981, pp. 189-195.

[12] F. HoRvath, An experimental comparison of the psychological stress evaluator and the galvanic skin response in detection of deception, J. Appl. Psychology (1978) 63 338-344.

[13] I. Kuroda, O. Fujiwara, N. Okamura, and N. Utsuki, Method for determining pilot stress trough analysis of voice communication, Aviation, Space and Environmental Medicine (1976) 47 528-533.

[14] G. Klasmeyer and W. Sendlmeier, Objective voice parameters to characterize the emotional content in speech, in: Proc. International Conference on Spoken Language Processing, 1995, 1, pp. 182-185.

[15] S. Mazziconacci, Pitch variations and emotion in speech, in: Proc. International Conference on Spoken Language Processing, 1995, 1, pp. 178-181.

[16] S. McGilloway, R. Cowie, and E. Douglas-Cowie, Prosodic signs of emotion in speech: Preliminary results from a new technique for automatic statistical analysis, in: Proc. International Conference on Spoken Language Processing, 1995 1, pp. 250-253.

[17] R. Ritz, C. Legos, And A. Guell, Voice analysis to predict the psychological state of a speaker, Aviation, Space and Environmental Medicine (1990) 61266 271.

[18] R. Ritz, E. Absil, B. Harmengies, C. Legos, and D. Poch, Time- and spectrum-related variabilities in stressed speech under laboratory and real condition, Speech Communication (1996) 20 111-129.

[19] R. Ritz AND C. LEgos, The cumulative spectral probability diagram: theory and experiments, Acta Acustica (1994) 4 215-222.

[20] H. Siegwart AND K. Scherer, Acoustic concomitants of emotional expression in opera singing, J. of Voice (1995) 9 249-260. 
[21] K. Scherer, Speech and emotional states, Speech Evaluation in Psychiatry (Ed. J. Derby) Grune \& Stratton, New York (1981).

[22] K. Scherer, Vocal indicators of stress, Speech evaluation in psychiatry (Ed. J. Derby) Grune \& Stratton, New York (1981).

[23] K. Scherer, How emotion is expressed in speech and singing, in: Proc. International Conference on Spoken Language Processing, 1995, 1, pp. 90-97.

[24] P. Simonov And V. Frolov, Analysis of the human voice as a method of controlling emotional state: Achievements and goals, Aviation, Space and Environmental Medicine (1977) 48 73-79.

[25] L. Streeter, N. MacDonald, W. Apple, R. Krauss, and K. Galotti, Acoustic and perceptual indicators of emotional stress, J. Acoust. Soc. Am. (1983) 73 1354-1360.

[26] J. Whitmore And S. Fisher, Speech during sustained operations, Speech Communication (1996) 20 (1-2) 55-70.

[27] C. Williams and K. Stevens, On determining the emotional state of pilots during flight: An exploratory study, Aerospace Medicine (1969) 40 1369-1372.

[28] C. Williams and K. Stevens, Emotions and Speech: Some acoustic correlates, J. Acoust. Soc. Am. (1972) 52 1238-1250.

[29] B. Womack and J. Hansen, Classification of speech under stress using driven features, Speech Communication (1996) 20 131-150.

[30] B. Womack And J. Hansen, Stressed speech classification with application to robust speech recognition, in: Proc. International Conference on Acoustics, Speech \& Signal Proc., 1996, pp. 53-56.

[31] F. Lacerda, Voice stress analyses: Science and pseudoscience, in: Proceedings of Meetings on Acoustics, May 2013, 19 (60003): 060003, doi:10.1121/1.4799435.

[32] Kristen Zambo, Statements in rape case allowed in, made 17 hours after voice stress test, judge rules, Journal Times (Racine, WI), (June 19, 2014), http://journaltimes.com/news/local/statements-in-rape-case-allowed-inmade-hours-after-. .

[33] Kelly R. Damphousse, Voice Stress Analysis: Only 15 Percent of Lies About Drug Use Detected in Field Test, National Institute of Justice Journal (March 16, 2008), https://nij.ojp.gov/topics/articles/voice-stress-analysis-only15-percent-lies-about-drug-use-detected-field-test

[34] James D. Harnsberger, Harry Hollien, Camilo A. Martin, and Kevin A. Hollien, Stress and Deception in Speech: Evaluating Layered Voice Analysis, J. Forensic Sci. (May 2009) 54 (3), doi: 10.1111/j.15564029.2009.01026.xAvailable online at: www.blackwell-synergy.com, https://www.kpu.edu.rs/data/materijali/bfi/Seminarski\%20radovi/Stress\%20 And\%20Deception\%20In\%20Speech\%20Evaluation\%20Layered\%20Voice\% 20Analysis.pdf 
[35] L. TAVI, Classifying females' stressed and neutral voices using acoustic-phonetic analysis of vowels: an exploratory investigation with emergency calls, Int. J. Speech Technol. (2019) 22 511-520, https://doi.org/10.1007/s10772-018-09574-6

[36] Vijay P. Patil, Krishna Kant Nayak, and Manish Saxena, Voice Stress Detection, International Journal of Electrical, Electronics and Computer Engineering (2013) 2 (2) 148-154, e-ISSN 2277-2626, https://sapavsa.co.za/wpcontent/uploads/2018/10/VSA-STUDY.pdf

[37] S. Hadjitodorov and B. Boyanov, Speaker recognition, Research report for the National Scientific Fund, February (1997) (in Bulgarian).

[38] B. Boyanov, S. Hadjitodorov, and G. Chollet, Robust periodicity/ aperiodicity detector, Ann. of Bulgarian Academy of Sciences (1997) 50 43-46.

[39] B. Boyanov, S. Hadjitodorov, T. Ivanov, and G. Chollet, Robust Hybrid Pitch Detector, Electronic Letters (1993) 29 (22) 1924-1926.

[40] L. Rabiner, R. Schaffer, Digital processing of speech signals, Prentice Hall, New Jersey (1978).

[41] H. Murthy, K. Murthy, and B. Yegnanarayana, Formant extraction from phase using weighted group delay function, Electronics Letters (1989) 251609 1611.

[42] H. NASHI, Phase unwrapping of digital signals, IEEE Trans. ASSP (1989) $\mathbf{3 7}$ $1693-1702$.

[43] G. Duncan, B. Yegnanarayana, and H. Murthy, A nonparametric method of formant estimation using group delay spectra, in: Proc. of Int. Conf. on ASSP, Dallas, USA, 1989, pp. 572-575.

[44] S. Hadjitodorov, B. Boyanov, T. Ivanov, and N. Dalakchieva, Textindependent speaker identification using neural nets and AR-models, Electronics Letters (1994) 30 (11) 838-840.

[45] S. Hadjitodorov, B. Boyanov, N. Dalakchieva, A two-level classifier for text-independent speaker identification, Speech Communication (1997) 21 (2) 209-217.

[46] T. Kohonen, The self-organizing map, Proc. IEEE (1990) 78 1464-1480.

[47] B. Boyanov and S. Hadjitodorov, Acoustic analysis of pathological voices and screening of laryngeal diseases, IEEE Engineering in Medicine $\&$ Biology (1997) 16 (4) 74-82.

[48] M. Gelfer, D. Fendel, Comparison of jitter, shimmer and SNR from directly digitized versus taped voice samples, J. of Voice (1995) 9 (4) 378-382.

Received December 08, 2020 